\title{
Bioanalysis
}

\section{Measuring and using free drug concentrations: has there been 'real' progress?}

\author{
"Although this area holds great promise for fine-tuning therapy and \\ reducing side effects from drugs, developments in the last 5 years \\ seem to be incremental only."
}

First draft submitted: 16 March 2017; Accepted for publication: 24 March 2017; Published online: 18 May 2017

Keywords: free drug concentration • pharmacological effect • total drug concentration

The determination of free concentrations has long been known to be important to a wide range of fields, from environmental science to anesthesiology. For therapeutics, the discovery that freely diffusible, or unbound, drug concentrations can be measured in biological samples has led to optimism regarding personalized drug therapy. However, with the exception of a few drugs and hormones, most therapeutic drug monitoring decisions continue to be based on total concentrations.

If free drug concentrations correlate with therapeutic effects better than total concentrations, as has been shown in numerous studies [1-4], why have they not been adopted more widely? The main reasons could be grouped in three categories: analytical, therapeutic and availability of funding for research.

On the analytical side, methods for measuring free drug concentrations not only suffer from lower precision and lower accuracy, but are also more time- and resourceintensive than methods for total concentrations. The higher measurement variability results from the lower values of these free concentrations, the more complicated sample preparation procedures and susceptibility to changes in equipment for processing, temperature, $\mathrm{pH}$ and dilution. While clinical laboratories usually measure total concentrations by immunoassays using minimally processed samples, methods for free concen- trations require atypical sample preparation equipment and are in need of more sensitive analytical instruments.

Regarding therapeutic decisions, although free concentrations are better correlated with pharmacological effects when the extent of plasma protein binding is highly variable or unknown, total concentrations are considered to be similarly useful when the free drug fraction does not change significantly between individuals. However, the constancy of the free drug fraction is only an assumption, as it has been shown numerous times that interindividual variability in protein binding ranges from almost no binding to very high binding. Unfortunately, this variability is usually ignored in clinical practice owing to lack of resources to address it. Furthermore, most clinicians consider that free concentrations offer only a modest improvement over total concentrations when it comes to adjusting drug dosage regimens.

Another significant hurdle for research into measurement and applications of free concentrations consists of limited availability of funding in this area. Funding decisions usually favor projects that promise highimpact transformative research; for example, proposals for developing new drugs have much better chances of being funded than proposals for reducing side effects of currently available drugs. In the case of research

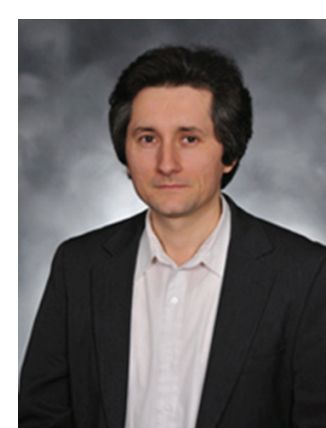

Florin Marcel Musteata Department of Pharmaceutical Sciences, Albany College of Pharmacy \& Health Sciences, 106 New Scotland Avenue, Albany, NY 12208, USA marcel.musteata@acphs.edu 
into free concentrations, the problem is exacerbated by the perceived minor benefit from using them instead of total concentrations. Accordingly, research into free concentrations continues to be a secondary area, with only a few laboratories around the world looking into the fundamental aspects of their measurement. Slightly more successful are the studies regarding applications of free concentrations, which result in several publications per year.

Since the last review of the methods available for measuring free concentrations [5], advances were made regarding the more sensitive determination of plasma protein binding for cationic drugs and ways to more accurately calibrate pre-equilibrium liquidphase microextraction; furthermore, several groups reported improved ultrafiltration-based approaches. Measurement of free concentrations for cationic drugs is particularly difficult since they have low partition coefficients in nonpolar extraction phases and their nonionized fraction in biological samples is low. To overcome these challenges, Peltenburg et al. used mixed-mode solid-phase microextraction based on octadecyl and propylsulfonic acid coating [6]. These fibers were found to be particularly well suited for quantifying the free concentration of several model cationic drugs, such as tramadol, amphetamine, amitriptyline and diazepam. The method was appropriate for determining drug-protein binding constants in simple mixtures, as well as for measuring the free drug fraction in plasma and whole blood samples. Albumin was found to bind to the coating to some degree, but this did not influence significantly the absorption of analytes. Although equilibrium extraction conditions are preferred for their higher accuracy, equilibration times can be in excess of $24 \mathrm{~h}$ for some drugs. In such cases, accurate methods for calibrating pre-equilibrium extractions are needed. In this regard, Xu et al. developed a label-free method for measuring the time constant in microextraction methods [7] and successfully applied it to determine the free concentration of flunitrazepam in biological fluids. Ultrafiltration is by far the most popular approach for measuring free concentrations, although it continues to be highly sensitive to experimental conditions; the impact of these conditions on the accuracy of free concentration measurements for vancomycin, ceftriaxone, cefazolin and ertapenem in plasma was investigated by Kratzer $e t a l$. [8]. The researchers found that the best reproducibility was obtained when the biological samples were maintained close to physiological conditions and were processed at low centrifugal forces. Another study by Li et al. confirmed that the value of the free fraction of vancomycin obtained by ultrafiltration changes dramatically and unexpectedly depending on the membrane material, the molecular weight cut off and the centrifugation conditions [9].

Besides technical developments, several interesting clinical applications of free concentrations were reported, ranging from new approaches for estimating drug doses in initial clinical trials to fine-tuning therapy in critically ill patients. During early drug discovery, protein binding is usually determined using affinity chromatography and is subsequently used in preliminary human trials in order to achieve efficient free concentrations at the targeted area [10]. The unbound drug concentrations and drug-target binding kinetics are also key parameters in formulating differential equations for $\mathrm{PK}-\mathrm{PD}$ modeling of in vivo activity, drug-drug interactions, influence of plasma protein binding on the volume of distribution and target-mediated drug disposition $[4,11,12]$. Free concentrations of antibiotics and antiepileptic drugs have long been known to be important for therapeutic effects, and the evidence keeps accumulating. Lana et al. have shown that increased free valproic acid in serum can induce its own metabolism, as well as that of other drugs, and therefore, decrease total concentrations in the body [13]. Excretion of valproic acid in saliva was found to be correlated with its free concentration in serum; therefore, measurement in saliva could be used as a noninvasive method for therapeutic monitoring of free concentrations in persons with epilepsy [14]. The area under the curve for unbound drug and the time when the unbound concentration is above the minimum inhibitory concentration were again confirmed to be important in treating several infectious diseases, such as those caused by Enterobacteriaceae and Mycobacterium tuberculosis [15,16].

So, has there been any progress in measuring and using free drug concentrations? Although, this area holds great promise for fine-tuning therapy and reducing side effects from drugs, developments in the last 5 years seem to be incremental only. Unless accurate bioanalytical methods for measuring free levels that are as fast and as affordable as those for total concentrations are developed, progress in individualizing drug therapy based on the concentration of freely diffusible molecules will likely continue to be modest.

\section{Financial \& competing interests disclosure}

The author has no relevant affiliations or financial involvement with any organization or entity with a financial interest in or financial conflict with the subject matter or materials discussed in the manuscript. This includes employment, consultancies, honoraria, stock ownership or options, expert testimony, grants or patents received or pending, or royalties.

No writing assistance was utilized in the production of this manuscript. 


\section{References}

1 Musteata FM. Making sense of vitamin D concentrations. Future Sci. OA 2(1), FSO90 (2016).

2 Musteata FM. The drug concentration conundrum. Analyt. Scientist 1115, 304 (2015).

3 Dasgupta A. Usefulness of monitoring free (unbound) concentrations of therapeutic drugs in patient management. Clin. Chim. Acta 377(1-2), 1-13 (2007).

4 Svennebring AM. The impact of the concentration of drug binding plasma proteins on drug distribution according to Oie-Tozer's model. Xenobiotica 46(4), 307-314 (2016).

5 Musteata FM. Monitoring free drug concentrations - challenges. Bioanalysis 3(15), 1753-1768 (2011).

6 Peltenburg H, Bosman IJ, Hermens JLM. Sensitive determination of plasma protein binding of cationic drugs using mixed-mode solid-phase microextraction. J. Pharm. Biomed. Anal. 115, 534-542 (2015).

7 Xu B, Hou J, Chen M, Chen X, Zhang X, Cui S. Calibration of pre-equilibrium HF-LPME and its application to the rapid determination of free analytes in biological fluids. J. Chromatogr. B 980, 28-33 (2015).

8 Kratzer A, Liebchen U, Schleibinger M, Kees MG, Kees F. Determination of free vancomycin, ceftriaxone, cefazolin and ertapenem in plasma by ultrafiltration: impact of experimental conditions. J. Chromatogr. B 961, 97-102 (2014).

9 Li X, Wang F, Xu B et al. Determination of the free and total concentrations of vancomycin by two-dimensional liquid chromatography and its application in elderly patients. J. Chromatogr. B 969, 181-189 (2014).

10 Teague $S$, Valko K. How to identify and eliminate compounds with a risk of high clinical dose during the early phase of lead optimisation in drug discovery. Eur. J. Pharm. Sci. doi:10.1016/j.ejps.2017.02.017 (2017) (Epub ahead of print).

11 Koch G, Jusko WJ, Schropp J. Target mediated drug disposition with drug-drug interaction, Part II: competitive and uncompetitive cases. J. Pharmacokinet. Pharmacodyn. 44(1), 27-42 (2017).

12 Vauquelin G. Cell membranes... and how long drugs may exert beneficial pharmacological activity in vivo. Br. J. Clin. Pharmacol. 82(3), 673-682 (2016).

13 Lana F, Marti-Bonany J, Fuster J, De LJ. Reduction in serum concentration of valproic acid secondary to the intake of ibuprofen as an example of valproic acid auto-induction metabolism. Actas Esp. Psiquiatr. 44(4), 136-144 (2016).

14 Dwivedi R, Gupta YK, Tiwari P et al. Correlation of saliva and serum free valproic acid concentrations in persons with epilepsy. Seizure 25, 187-190 (2015).

15 Alsaad N, Dijkstra JA, Akkerman OW et al. Pharmacokinetic evaluation of sulfamethoxazole at 800 milligrams once daily in the treatment of tuberculosis. Antimicrob. Agents Chemother. 60 (7), 3942-3947 (2016). Laterre P-F, Wittebole X, Van DVS et al. Temocillin ( $6 \mathrm{~g}$ daily) in critically ill patients: continuous infusion versus three times daily administration. J. Antimicrob. Chemother. 70(3), 891-898 (2015). 